\title{
Fine specificity of antibodies against AQP4: Epitope mapping reveals intracellular epitopes
}

\author{
E.I. Kampylafka ${ }^{\text {a }}$, J.G. Routsias ${ }^{\text {a }}$, H. Alexopoulos ${ }^{\text {a }}$, M.C. Dalakas ${ }^{\text {a,b }}$, \\ H.M. Moutsopoulos ${ }^{a}$, A.G. Tzioufas ${ }^{\mathrm{a}, *}$ \\ a Department of Pathophysiology, Medical School, National and Kapodistrian University of Athens, 75 Mikras Asias str., 11527 Athens, Greece \\ ${ }^{\mathrm{b}}$ Department of Neurology, Thomas Jefferson University, Philadelphia, PA, USA
}

\section{A R T I C L E I N F O}

\section{Article history:}

Received 22 December 2010

Received in revised form

14 January 2011

Accepted 18 January 2011

\section{Keywords:}

Autoantibodies

Neuromyelitis optica

Epitope mapping

Molecular mimicry

\begin{abstract}
A B S T R A C T
The autoantibody to aquaporin-4 (AQP4) is a marker and a pathogenetic factor in Neuromyelitis Optica (NMO) (Devic's syndrome). Our aim was to identify B-cell antigenic linear epitopes of the AQP4 protein and investigate similarities with other molecules. To this end, we screened sera from 21 patients positive for anti-AQP4 antibodies (study group), from 23 SLE and 23 pSS patients without neurologic involvement (disease controls) and from 28 healthy individuals (normal controls). Eleven peptides, spanning the entire intracellular and extracellular domains of the AQP4 molecule, were synthesized, and all sera were screened for anti-peptide antibodies by ELISA. Specificity was evaluated by homologous inhibition assays. NMO positive sera exhibited reactivity against 3 different peptides spanning the sequences aa1-22 (AQPpep1) (42.9\% of patients), aa88-113 (AQPpep4) (33\%) and aa252-275 (AQPpep8) (23.8\%). All epitopes were localized in the intracellular domains of AQP4. Homologous inhibition rates were ranging from $71.1 \%$ to 84.3\%. A 73\% sequence homology was observed between AQPpep8' aa257-271, a 15-mer peptide part of the AQPpep8 aa252-275, and the aa219-233 domain of the Tax1-HTLV-1 binding protein (TAX1BP1), a host protein associated with replication of the Human T-Lymphotropic Virus 1 (HTLV-1). Antibodies against the AQP4 and the TAX1BP1 15-mer peptides were detected in $26.3 \%(N=5)$ and $31.6 \%(N=6)$ of NMO positive sera $\left(r_{\mathrm{s}}=0.81, P<0.0001\right)$. Healthy controls did not react with these peptides, while homologous and cross-inhibition assays confirmed binding specificity. This first epitope mapping for AQP4 reveals that a significant proportion of anti-AQP4 antibodies target linear epitopes localized in the intracellular domains of the channel. One of the epitopes displays high similarity with a portion of TAX1BP1 protein.
\end{abstract}

() 2011 Elsevier Ltd. All rights reserved.

\section{Introduction}

Neuromyelitis optica (NMO or Devic's Disease) is a rare autoimmune inflammatory demyelinating syndrome of the Central Nervous System (CNS) that preferentially targets the optic nerves and spinal cord [1]. Its course is usually relapsing, but without marked remission between relapses, leading to rapid accumulation of irreversible deficits [2]. The studies by Lennon et al. [3] have provided unequivocal evidence that a specific autoantibody (NMO$\operatorname{IgG}$ ) against aquaporin-4 (AQP4) is a marker for the disease and differentiates neuromyelitis optica from multiple sclerosis [1]. Further studies have shown that anti-AQP4 antibodies are not only detected in neuromyelitis optica, but also in patients with the "NMO spectrum of diseases" [4] that includes limited forms of NMO

\footnotetext{
* Corresponding author. Tel.: +30 2107462512; fax: +30 2107462664.

E-mail address: agtzi@med.uoa.gr (A.G. Tzioufas).
}

[5], optic-spinal MS [6], and demyelinating conditions accompanying systemic autoimmune diseases, such as Systemic Lupus Erythematosus and Sjögren's Syndrome [5].

Aquaporin- 4 belongs to a family of water channels (AQP0-12) involved in water transport in different tissues [7]. It consists of 3 extracellular loops, 2 intracellular loops and the $\mathrm{N}$ - and C-terminal regions of the protein, that connect 6 alpha helices spanning the membrane [8]. It exists as heterotetramers composed by the $\mathrm{M}_{1}$ (M.W. $34 \mathrm{kDa}$ ) and $\mathrm{M}_{23}$ (M.W. $31 \mathrm{kDa}$ ) splice variants of aquaporin4 , which differ in their first 22 amino acids [9]. AQP4 is the most abundant water channel in the CNS and is highly expressed on the astrocytic endfeet at the blood-brain barrier (BBB), regulating water transport between blood, brain and cerebrospinal fluid [10].

Autoantibodies to AQP4 appear to possess a pathogenetic role, as shown by previous studies, since they induce surface redistribution and endocytosis of AQP4, complement activation, water homeostasis and blood-brain barrier disruption, leading eventually to the recruitment of inflammatory cells [11]. 
Furthermore, NMO-like disease was successfully induced after passive transfer in animal models, following BBB breakdown $[12,13]$, or by direct intra-cerebral IgG injection [14].

Despite the fact that anti-AQP4 antibodies have an established role in the pathogenesis of neuromyelitis optica, their specificity regarding antigenic epitopes has not yet been determined. Some studies support the existence of conformation-dependent epitopes $[15,16]$, although others suggest the recognition of linear epitopes in the denatured molecule [17]. The aim of the present study was to identify the B-cell linear epitopes of the AQP4 protein and to investigate antigenic similarities with other molecules.

\section{Materials and methods}

\subsection{Patients and sera}

We used sera from 21 patients positive for NMO IgG/anti-AQP4 antibodies, as detected by indirect immunofluorescence on mouse brain tissue [3] (21/21 positive) and by a cell based assay using $\mathrm{M}_{23}$ transfected $\left(\mathrm{M}_{23}-\mathrm{eGFP}\right.$ clones were a kind gift from Dr. P. Waters and Prof. A. Vincent, University of Oxford) HEK293 cells [18] (16/21 positive). All patients belonged to the neuromyelitis optica spectrum of diseases [4] and presented active CNS involvement during blood sampling, as attested by the clinical picture and MR Imaging. Sera from 28 healthy subjects were used as normal controls. The disease control group comprised of 23 patients with Systemic Lupus Erythematosus (SLE) and 23 patients with primary Sjögren's Syndrome (pSS), without neurological involvement. All SLE patients fulfilled the 1997 American College of Rheumatology revised criteria for the classification of Systemic Lupus Erythematosus [19], while the pSS patients fulfilled the AmericanEuropean Consensus Group revised criteria [20].

\subsection{Peptide synthesis}

Eleven peptides, in the form of peptide dendrimers (multiple antigenic peptides, MAP) were synthesized by Bio-synthesis Inc, U.S.A. The peptides covered all the intracellular and extracellular domains of both AQP4 isoforms $\left(\mathrm{M}_{1}\right.$ : UniProtKB accession number P55087-1, $\mathrm{M}_{23}$ : UniProtKB accession number P55087-2), and were either single peptides or overlapping by 8 amino acids. The first 2 peptides (AQPpep1 and AQPpep2) covered the N-terminus intracellular domain of the protein (Fig. 1). AQPpep1 was designed to include the first 22 amino acids that are included only in the $M_{1}$ isoform of the protein in a non-overlapping fashion with AQPpep2 that starts exactly at the $\mathrm{N}$-terminus of $\mathrm{M}_{23}$ isoform. Peptides AQPpep3-AQPpep7 corresponded to the three extracellular and two intracellular loops of the protein and, finally, the C-terminus intracellular domain of the protein was covered by four 24-mer peptides, overlapping by 8 amino acids (Peptides AQPpep8,
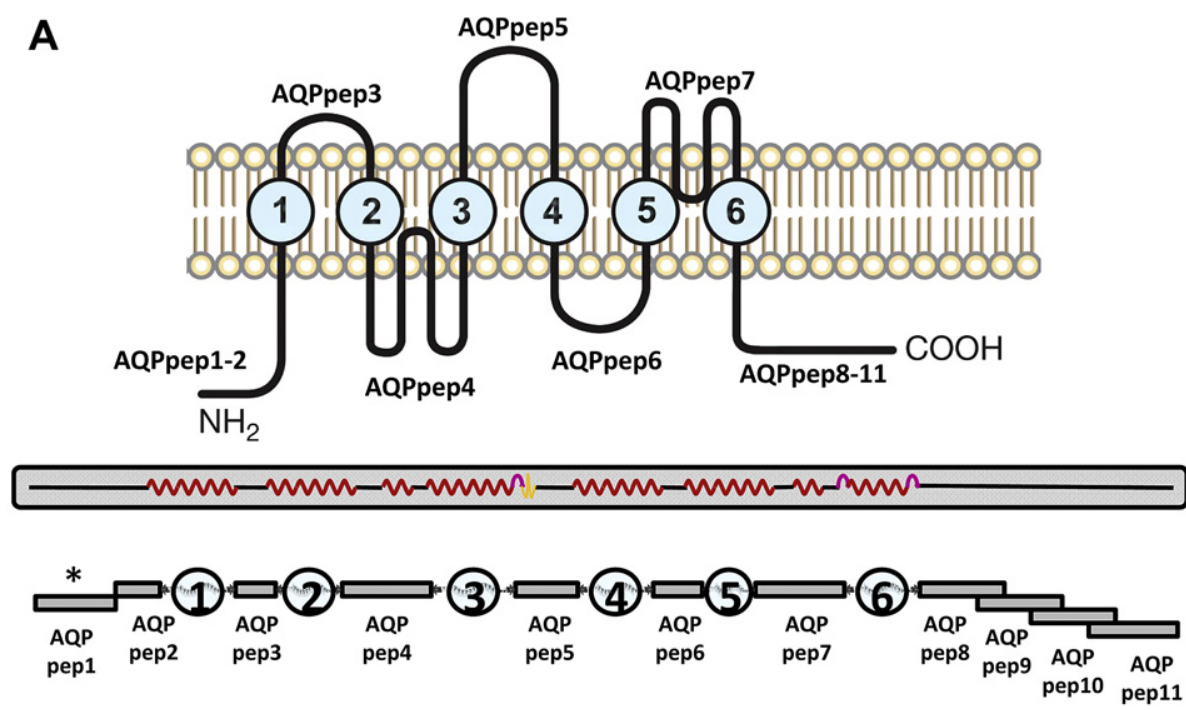

B

\begin{tabular}{llll}
\hline Peptide name & Location & Sequence & Amino acids \\
\hline AQPpep1 & intracellular & MSDRPTARRWGKCGPLCTRENI & [aa1-22] \\
AQPpep2 & intracellular & MVAFKGVWTQAFWK & [aa23-36] \\
AQPpep3 & extracellular & NWGGTEKPLPVDM & [aa57-69] \\
AQPpep4 & intracellular & FGHISGGHINPAVTVAMVCTRKISIA & {$[$ aa88-113] } \\
AQPpep5 & extracellular & TPPSVVGGLGVTMVHGNLT & {$[$ aa137-155] } \\
AQPpep6 & intracellular & SCDSKRTDVTGSIA & {$[$ aa177-190] } \\
AQPpep7 & extracellular & INYTGASMNPARSFGPAVIMGNWENH & {$[$ aa205-230] } \\
AQPpep8 & intracellular & FCPDVEFKRRFKEAFSKAAQQTKG & {$[$ aa252-275] } \\
AQPpep9 & intracellular & KAAQQTKGSYMEVEDNRSQVETDD & {$[$ aa268-291] } \\
AQPpep10 & intracellular & RSQVETDDLILKPGVVHVIDVDRG & {$[$ aa284-307] } \\
AQPpep11 & intracellular & HVIDVDRGEEKKGKDQSGEVLSSV & {$[$ aa300-323] } \\
\hline
\end{tabular}

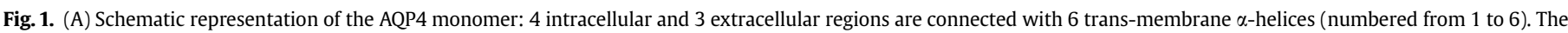

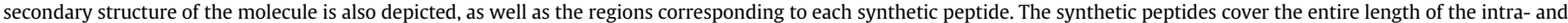

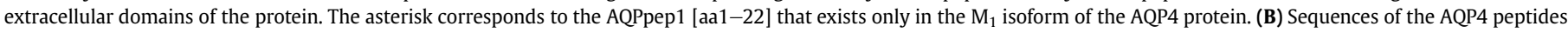
and their exact location within the protein are shown. 
AQPpep9, AQPpep10 and AQPpep11). Two additional 15-mer peptides corresponding to the [aa257-271] of the AQP4 protein and the [aa219-233] of the TAX1BP1 (Tax1-HTLV-1 binding protein, UniProtKB accession number Q86VP1) were also synthesized (Table 1).

\subsection{ELISA assays}

All patients and controls were evaluated for the presence of autoantibodies against the 11 peptides by ELISA assays. 96-well microtitre plates were coated with $100 \mu \mathrm{l}$ of peptide solution $(2.5 \mu \mathrm{g} / \mathrm{ml}$ in sodium carbonate-sodium bicarbonate buffer, PH 9.8) and kept at $4{ }^{\circ} \mathrm{C}$ overnight. Afterwards, the remaining binding sites were blocked with $2 \%$ bovine serum albumin (BSA) in Phosphate Buffered Saline (PBS) at room temperature for $1 \mathrm{~h}$. After 3 washes with PBS, sera were added in $1 / 150$ in $2 \%$ BSA/PBS dilution. After a 2-h incubation and 3 washes with PBS, alkaline phosphate conjugated anti-human IgG, diluted $1 / 1200$ in $2 \%$ BSA/PBS, was added for $1 \mathrm{~h}$ at room temperature. After the addition of alkaline phosphate substrate, color development was quantified at $405 \mathrm{~nm}$. The cutoff values for each peptide assay were determined using the mean Optical Density (OD) plus 3 standard deviations of the sera from the 28 healthy controls.

Homologous inhibition experiments were performed, in order to evaluate the specificity of the potential epitopes. Sera from 3 NMO positive patients, as well as a pSS and an SLE patient, that exhibited high reactivity against the peptides, were selected. Serum was pretreated in $1 / 300$ dilution with increasing concentrations (0 $\mu \mathrm{g} / \mathrm{ml}, 10 \mu \mathrm{g} / \mathrm{ml}$ and $40 \mu \mathrm{g} / \mathrm{ml}$ ) of peptides AQPpep1, AQPpep4, AQPpep8 (being the 3 most reactive peptides) and control peptide (Ctrl-Pep), and incubated overnight at $4{ }^{\circ} \mathrm{C}$. Peptide AQPpep9 was chosen as a control, since it exhibited low reactivity during the first series of experiments. Subsequently, all sera dilutions were tested by ELISA for reactivity against the peptides. Inhibition percentages were calculated as $(([\mathrm{OD} 0 \mu \mathrm{g} / \mathrm{ml}]-[\mathrm{OD} 40 \mu \mathrm{g} / \mathrm{ml}]) /$ $[\mathrm{OD} 0 \mu \mathrm{g} / \mathrm{ml}]) \times 100 \%$ for each serum. All the steps of the assay were as described in Section (2.3), apart from the incubation of the sera dilutions that lasted $1 \mathrm{~h}$ instead of 2 .

\subsection{Homology search}

The antigenic peptide sequences were compared against the UniProtKB database (version 2010_10). The similarity search was performed using the NCBI BLASTP (ver. 2.2.17) algorithm and scored with PAM30 matrices. A 73\% sequence similarity was observed between AQPpep8 (amino acids 257-271) EFKRRFKEAFSKAAQ and the aa219-233 domain of the human protein TAX1BP1 (EFKKRFSDATSKAHQ) (Table 1). None of the other peptides showed any similarity with non-related to aquaporin human, bacterial or viral proteins.

2.5. ELISA assays to examine the potential cross-reactivity between AQP4 and TAX1BP1 15-mer peptides and heterologous inhibition assays

19 NMO positive patients and 19 normal controls were tested for the binding of autoantibodies against the two 15-mer peptides

Table 1

Homologous sequences of the AQP4 and TAX1BP1 15-mer peptides.

\begin{tabular}{lll}
\hline Origin & Sequence & Amino acids \\
\hline AQPpep8 (AQP4 protein) & EFKRRFKEAFSKAAQ & [aa6-20] \\
& EFK RF +A SKA Q & \\
TAX1BP1 protein & EFKKRFSDATSKAHQ & [aa219-233] \\
\hline
\end{tabular}

“+” symbol indicates conservative changes in amino acid sequence.
(AQPpep8' [aa257-271] of the AQP4 protein and TAX1BP1pep [aa219-233] of the TAX1BP1) on plates coated with $5 \mu \mathrm{g} / \mathrm{ml}$ of each peptide in sodium carbonate-sodium bicarbonate buffer, $\mathrm{PH} 10.5$. ELISA was performed as described above, Section (2.3). In order to investigate whether the two 15 -mer peptides could cross-inhibit one another, a second series of inhibition assays was performed in liquid phase. 3 selected NMO positive sera that exhibited high reactivity against the 2 peptides were pretreated in dilution 1/400 overnight at $4{ }^{\circ} \mathrm{C}$ with increasing concentrations of each peptide separately, as well as control AQPpep9 $(0 \mu \mathrm{g} / \mathrm{ml}, 10 \mu \mathrm{g} / \mathrm{ml}$, $40 \mu \mathrm{g} / \mathrm{ml}$ ). Subsequently, all sera were tested by ELISA as in Section (2.3), with the sole exception of incubation of the sera dilutions that lasted 50min.

\subsection{Statistical analysis}

All comparisons between groups of sera were performed using Fisher's exact test.

Spearman's rank correlation coefficient $\left(r_{\mathrm{s}}\right)$ was used to assess statistical dependence between reactivity rates of NMO positive sera against the two 15-mer peptides.

\section{Results}

\subsection{Identification of the antigenic sites and inhibition assays}

Peptides AQPpep1, 2, 4, 5 and 8 were identified as being the most reactive ones in an initial epitope mapping experiment in which all 11 peptides were tested. The ELISA assay was performed using sera from ten NMO positive patients and eight healthy controls. Sera from healthy donors showed no reactivity against any of the peptides. Subsequently, all sera from NMO positive patients $(N=21)$ and healthy controls $(N=28)$ were tested against the peptides, which exhibited the highest reactivity in the initial experiment (AQPpep1, 2, 4, 5 and 8). Peptides AQPpep1, AQPpep4 and AQPpep8 contained the epitopes that were mostly recognized by the NMO positive sera, reaching reactivity levels of $42.9 \%, 33.3 \%$ and $23.8 \%$ of patients, respectively (Fig. $2 \mathrm{~A}$ ). 5 sera (23.8\%) were positive for antibodies against all 3 peptides, 2 (9.5\%) were positive for antibodies against peptides 1 and 4, and 2 more sera were positive for antibodies only against peptide 1 .

The 3 most reactive peptides (AQPpep1, AQPpep4 and AQPpep8), are part of the intracellular regions of the molecule (Fig. $2 \mathrm{~B}$ ). These peptides were further tested against 23 SLE and 23 pSS disease controls, in order to assess the specificity of their recognition. SLE patients were found positive for antibodies against peptides AQPpep1, AQPpep4 and AQPpep8 in 13\%, $4.3 \%$ and $8.7 \%$, respectively, while $8.7 \%, 4.3 \%$ and $8.7 \%$ of pSS patients also recognized peptides AQPpep1, AQPpep4 and AQPpep8.

The proportion of NMO positive sera binding against peptides AQPpep1, AQPpep4 and AQPpep8 was statistically significantly higher than the proportion in healthy control individuals $(P<0.05)$. Antibodies against AQPpep1 and AQPpep4 also binded in a statistically significant manner in comparison to sera from disease controls (Fig. 3). The specificity of binding was assessed by homologous inhibition assays, which produced high inhibition rates in NMO positive sera that reached $84.3 \%, 71.1 \%$ and $84 \%$ for peptides AQPpep1, AQPpep4 and AQPpep8, respectively. Average inhibition rates, at $40 \mu \mathrm{g} / \mathrm{ml}$ of inhibitor, were $71.2 \%$ for AQPpep1, $67.9 \%$ for AQPpep4 and $66.9 \%$ for AQPpep8. On the other hand, inhibition rates with the control peptide (AQPpep9) were significantly lower, ranging from $11 \%$ to $45.3 \%$ (Fig. 4). Inhibition assays using disease control sera showed minimal inhibition rates, ranging from $3 \%$ to $15.1 \%$, that were similar to the ones produced when the control peptide was used (3.1-9.3\%). 
A
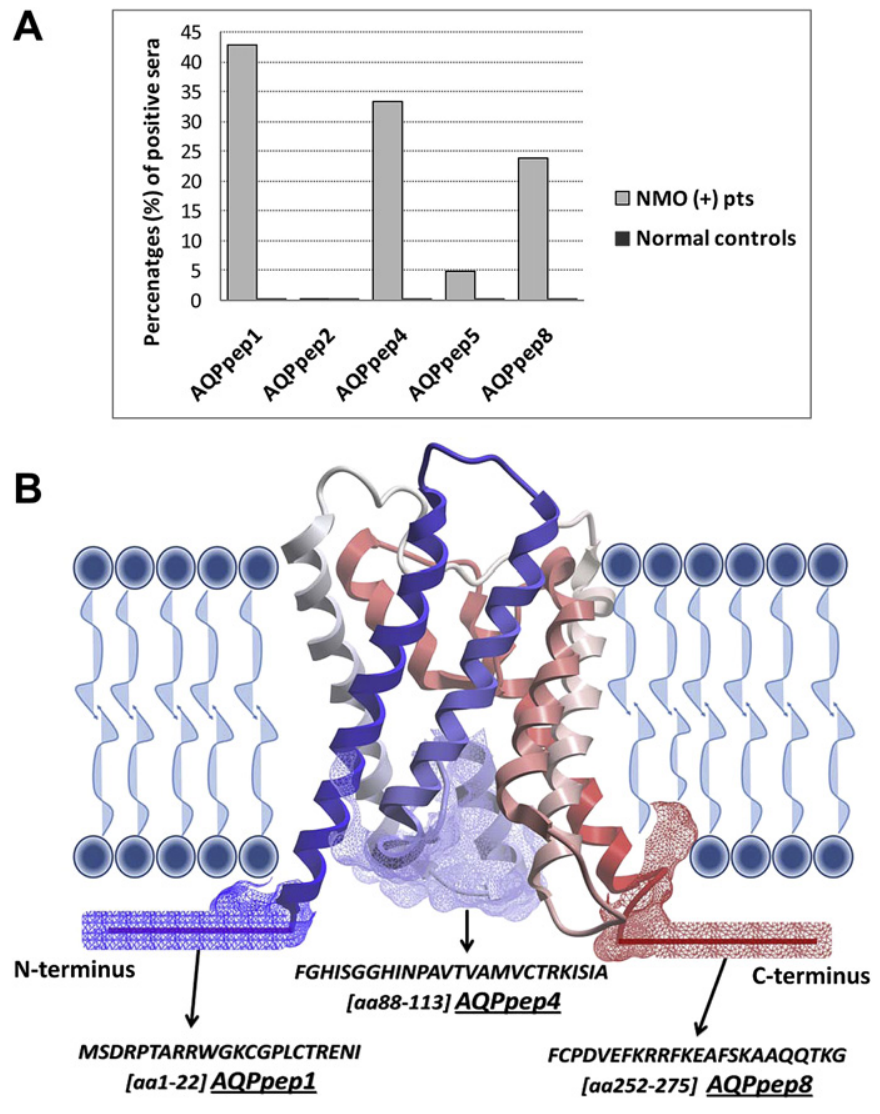

Fig. 2. (A) Prevalence of the NMO positive and healthy control sera who were positive for antibodies against peptides AQPpep1, AQPpep2, AQPpep4, AQPpep5 and AQPpep8. (B) The exact location of the 3 immunodominant AQP4 peptides on the tertiary structure of the AQP4 protein is shown. The three-dimensional structure of the molecule is represented as ribbons, whose color is gradually changing from blue (at the N-terminus) to red (at the C-terminus of the protein). The tertiary structure of the $\mathrm{N}$ - and C-terminus of AQP4 (aa1-30 and aa255-323, respectively) has not yet been solved and is therefore represented as two boxes containing straight lines. (For interpretation of the references to colour in this figure legend, the reader is referred to the web version of this article.)

\subsection{Sequence similarities of the immunodominant epitopes}

The identification of the 3 most reactive peptides, which are therefore potential linear antigenic epitopes of the AQP4 molecule, enabled the search of protein databases to identify similar sequences with other unrelated proteins. A 73\% sequence similarity was observed between AQPpep8 (amino acids 257-271) EFKRRFKEAFSKAAQ and the [aa219-233] domain of the human protein TAX1BP1 (EFKKRFSDATSKAHQ) (Table 1), which is involved in the replication of the HTLV-1 virus, the etiological agent of HTLV1 -associated myelopathy/tropical spastic paraparesis (HAM/TSP).

\subsection{Reactivity of the patients' sera against the AQP4 and the TAX1BP1 15-amino acid peptides and inhibition experiments}

These observations prompted us to design two 15-mer peptides containing the previously mentioned sequences (Table 1 ), and test them against the NMO positive sera and sera from healthy controls. Reactivity against the AQPpep8' and the TAX1BP1pep peptide was detected in $26.3 \%(N=5)$ and $31.6 \%(N=6)$ of NMO positive sera, respectively. All NMO-IgG-positive sera that were positive for antibodies against the AQPpep8', were also positive for antibodies against the TAX1BP1pep, while one patient was positive for antibodies against the TAX1BP1pep (Fig. 5A). Spearman's rank
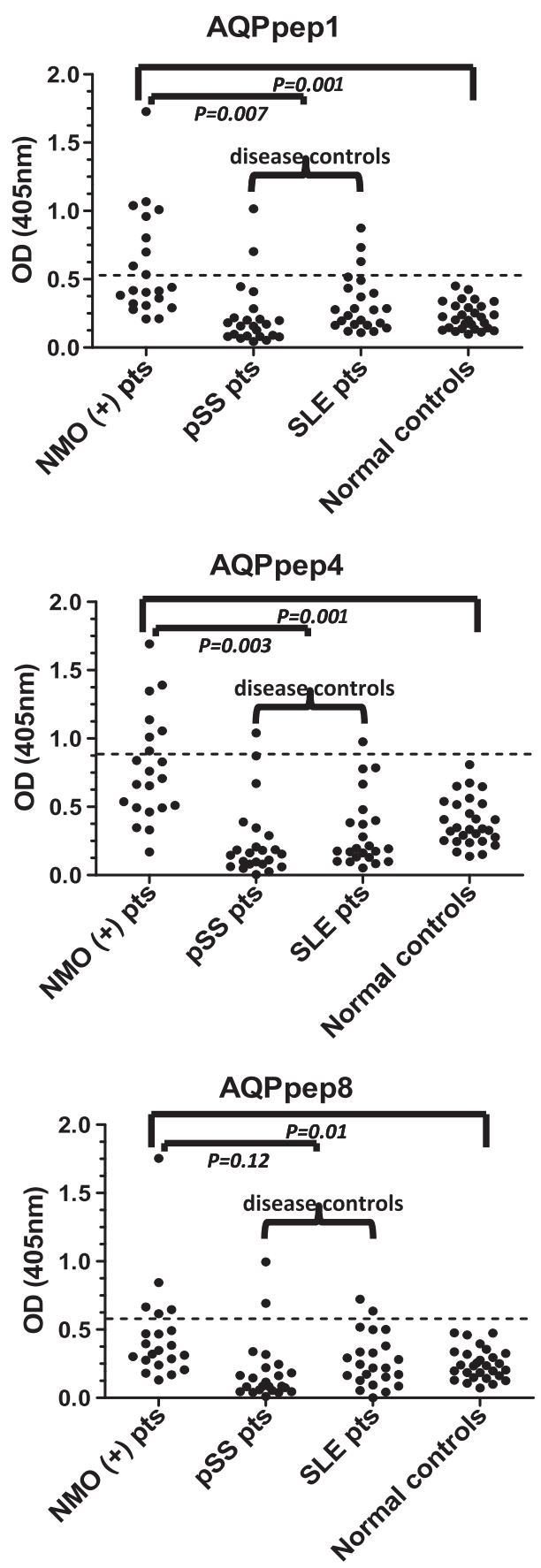

Fig. 3. Prevalence of positivity against peptides AQPpep1, AQPpep4 and AQPpep 8 for all groups (NMO patients, disease controls and healthy controls) is presented. Dotted lines represent the cutoff value for each peptide that was determined using the mean Optical Density (OD) plus 3 standard deviations of the sera from the 28 healthy controls. The statistical significance of comparisons between NMO positive sera and disease control sera, as well as between NMO positive and normal sera, is also presented (cutoff for significance $P<0.05$ ).

correlation coefficient was high $\left(r_{\mathrm{s}}=0.081, P<0.0001\right)$. None of the normal controls exhibited any reactivity against any of the 2 peptides.

The potential of anti-AQPpep $8^{\prime}$ antibodies to cross-recognize the TAX1BP1 pep was explored by homologous and cross-inhibition experiments. Pretreatment of the NMO positive sera with AQPpep ${ }^{\prime}$ and TAX1BP1pep resulted in a $60.1 \%$ and $36 \%$ inhibition of antibody binding to the AQPpep8' peptide (homologous and heterologous 

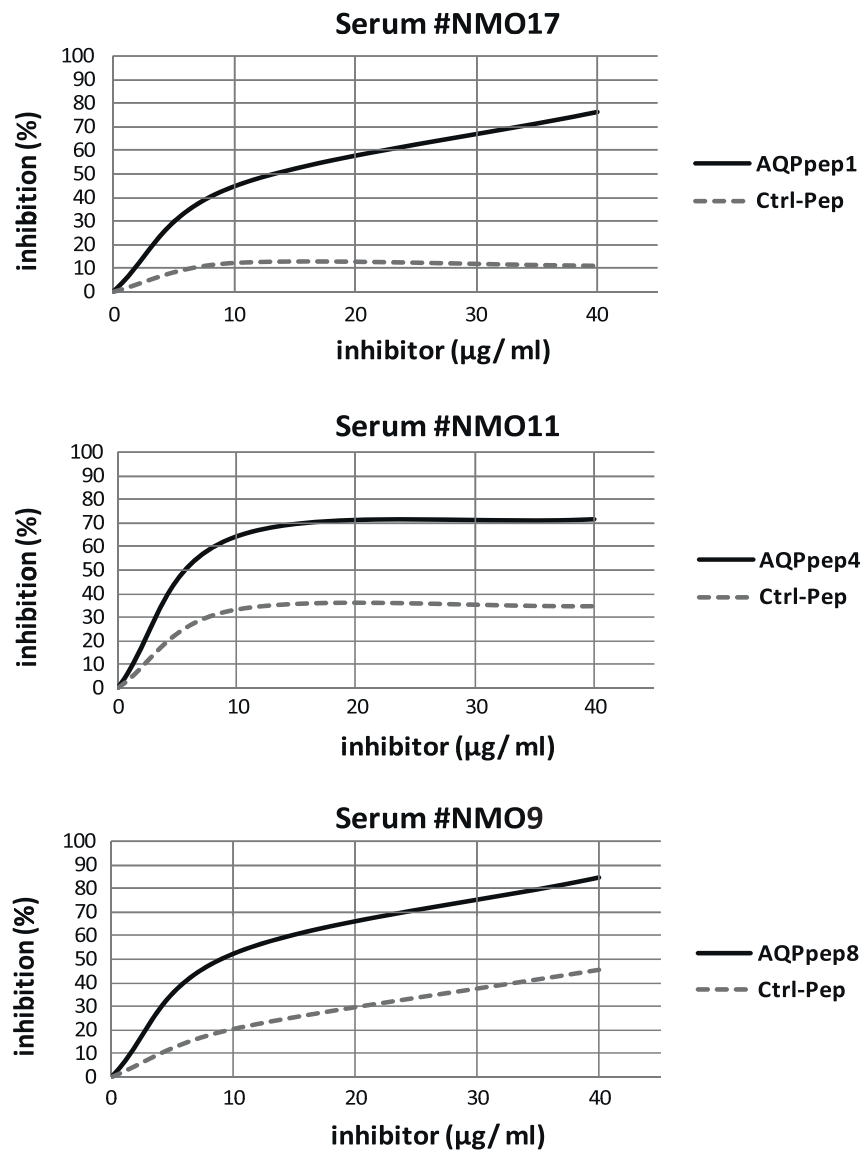

Fig. 4. Homologous inhibition assays with each specific peptide produced high inhibition rates while pretreatment with the control peptide (Ctrl-Pep), led to minimal inhibition rates. 3 representative patients are depicted in the figure (with inhibition rates ranging from $71.1 \%$ to $84.3 \%$ ).

inhibition, respectively). On the other hand, binding to TAX1BP1pep, was reduced by $70 \%$ after pretreatment with the TAX1BP1pep, and by $58.5 \%$ after pretreatment with AQPpep $8^{\prime}$. By contrast, control peptide (AQPpep9) did not inhibit AQPpep8' and TAX1BP1 pep binding (Fig. 5B).

\section{Discussion}

Since the discovery of anti-AQP4 antibodies as markers and pathogenetic factors in NMO, a great variety of methods have been developed for measuring anti-AQP4 antibodies in NMO patients with differences in sensitivity and specificity. This diversity denotes differences to the nature of the antigen used for detection.

The original and most commonly used detection method is the indirect immunofluorescence assay (IIF) against mouse brain sections in which the antigen is presumably in its native form [3]. Certain studies report that the autoantibodies target epitopes on the extracellular regions of the AQP4 molecule in a conformationdependent manner, since they recognize AQP4 only in the form of orthogonal arrays of particles (OAPs) [15]. In other studies, using ELISA and western blots that utilize denatured proteins as substrates, it is linear structures that are mainly detected [17]. Therefore, it is highly likely that both conformational and linear epitopes are targets of a polyclonal response against the aquaporin4 autoantigen, as described for the majority of the autoantibodies [21].

In order to identify the fine specificity of autoantibodies targeting aquaporin-4, we used overlapping synthetic peptides
A

Reactivity of NMO positive sera

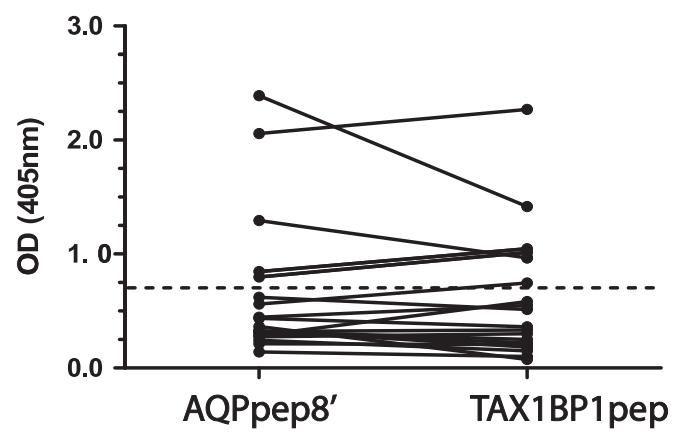

B
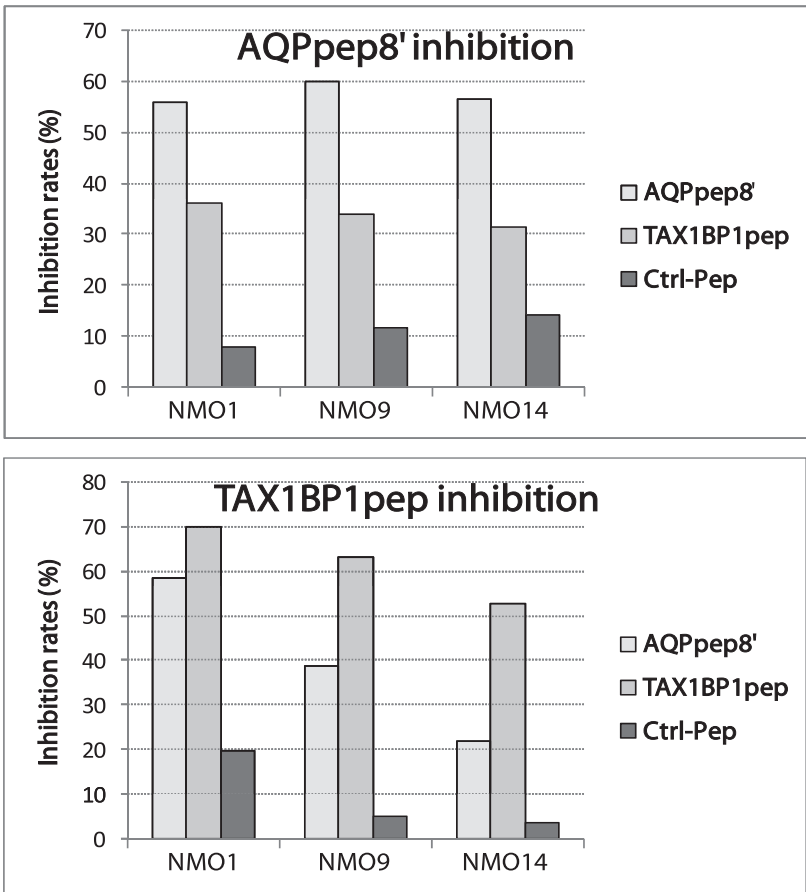

Fig. 5. (A) Reactivity levels of NMO positive sera are similar between peptides AQPpep8' and TAX1BP1pep (Spearman's rank correlation coefficient $\left(r_{s}\right)=0.81$, $P<0.0001$ ). (B) Homologous and heterologous inhibition assays reveal cross-reactivity of the two 15-mer peptides. Homologous inhibition rates were higher than heterologous ones for both peptides, while pretreatment with Ctrl-Pep did not inhibit either AQPpep8' or TAX1BP1pep binding. (Concentration of the inhibitor was $40 \mu \mathrm{g} / \mathrm{ml}$ ).

covering all the extracellular and intracellular domains of the molecule. Such peptides can effectively mimic linear and partially conformational epitopes and have been used in the past to map the specificity of autoantibodies found in different autoimmune diseases, including anti-Sm [22], anti-U1 snRNP [23], anti-Ro/SSA [24], anti-La/SSB [25], antibodies against citrullinated a-enolase [26], and against anti-glutamic acid decarboxylase (GAD) 65 [27]. 11 peptides were synthesized, covering all the intracellular and extracellular potential binding sites of the anti-AQP4 antibodies. Screening the NMO-IgG-positive sera for antibodies against these peptides, identified 3 dominant epitopes on the $\mathrm{N}$-terminus, the first intracellular loop and the C-terminus part of the protein. Disease control sera exhibited only minimal reactivity against the peptides. This reactivity can be attributed to humoral polyreactivity commonly observed in the sera of patients with autoimmune diseases. As $42.9 \%$ of the NMO positive sera recognized at least one of the 3 peptides, a large body of specific autoantibodies is probably 
directed against linear epitopes of the AQP4 protein. The inhibition assays, verified the specificity of the recognition.

Interestingly, the most reactive of the 3 epitopes corresponded to the first 22 amino acids of the AQP4, which exist only in the $M_{1}$ and not in the $\mathrm{M}_{23}$ isoform of the protein, suggesting that the $\mathrm{M}_{1}$ isoform exhibits definite antigenic sites. This finding agrees with Marnetto et al. [17] who showed, using western blot, that the major target of anti-aquaporin-4 antibodies in NMO is the linear AQP4-M isoform. Our observation also supports the recent finding of two different missense allelic mutations at Arg19 (R19I and R19T), which enhance susceptibility to NMO and are located within the first 22 residues of the $\mathrm{N}$-terminus of the protein, unique to the AQP4- $M_{1}$ [28]. The $M_{1}$ isoform of AQP4 seems to be important for NMO, since it is preferentially expressed in the optic nerve and spinal cord, where NMO lesions are usually found [29]. However, in another study, the $\mathrm{M}_{23}$ isoform has been proposed as a major target of anti-aquaporin-4 antibodies [15]. In this report an OAP-related, quaternary structure epitope, was identified on the surface of living cells selectively transfected to express $\mathrm{M}_{23}$ isoform. Since native aquaporin- 4 assemblies as heterotetramers of $M_{1}$ and $M_{23}$ splice variants forming intermediate size OAPs, the artificial enlargement of the OAPs caused by the over-expression of $\mathrm{M}_{23}$ isoform could enhance the avidity of autoantibody binding due to their potential to allow the bivalent binding of autoantibodies. Although this can be an advantage for the diagnostic detection of anti-AQP4 antibodies, it does not imply that all NMO autoantibodies bind only $\mathrm{M}_{23}$.

All 3 B-cell epitopes identified in our study were surprisingly located in the intracellular domains of the molecule. The mechanisms of recognition of intracellular epitopes are more complex, and to a great extend unknown. Many autoantibodies directed against intracellular antigens, including anti-U1RNP, anti-dsDNA, anti-Ro/SSA, anti-La/SSB, anti-Hu and others, have the potential to penetrate cells [30-33], in vivo [34,35]. Alternatively, the priming of the autoimmune response can follow apoptosis and release of intracellular epitopes or internalization. In the case of NMO, the polyclonal response we describe could be following astrocytic damage. A recent study was able to pinpoint $\mathrm{CD} 4^{+} \mathrm{T}$-cell epitopes of the AQP4 protein in its intracellular domains (in C57BL/6 and SJL/J mice) [36], suggesting that T-cell assistance may be also necessary to mount an efficient immune response against intracellular fragments of aquaporin. In contrast to our finding, Hinson et al. [10], based on the observation that anti-AQP4 antibodies bind to the surface of AQP4-transfected cells in the absence of conditions allowing membrane permeability, proposed that the epitopes are located in the extracellular regions of the protein. Several other studies have also focused only on the extracellular regions rather than the intracellular domains of aquaporin-4. Tani et al. [16], constructed amino acid substitution mutants in the extracellular domains of mouse $\mathrm{M}_{23}$-AQP4 and after comparisons of immunostaining intensity between human wild-form, mouse and rat AQP4, suggested that the third extracellular loop (E-loop) of AQP4 is a major epitope for the AQP4 antibodies.

A search of protein databases led to the detection of a $73 \%$ sequence similarity between AQP4 [aa257-271] and a 15-mer peptide from TAX1BP1 protein. TAX1BP1 is a human protein involved in Human T-cell Leukemia Virus type 1 (HTLV-1) replication $[37,38]$. HTLV-1 is the causative agent of HTLV-1-associated myelopathy or tropical spastic paraparesis (HAM/TSP) [37], a condition that shares some common clinical characteristics with neuromyelitis optica. A putative association between HTLV-1 infection and NMO has been previously reported by two study groups, which showed active HTLV-1 infection related to longitudinal extensive transverse myelitis (LETM, an NMO spectrum disorder) accompanied by anti-AQP4 antibodies [39].
In our study, NMO positive sera were examined for their reactivity against the two homologous 15-mer peptides (derived from AQP4 and TAX1BP1, respectively), revealing a high concordance of positivity. The specificity of the reaction as evaluated by the ability of the 2 peptides to cross-inhibit one another led to high inhibition rates for both peptides. Whether the cross-reactivity of anti-AQP4 antibodies with domains of the TAX1BP1 protein suggests a potential link between NMO and neurotropic retroviruses or it is an epiphenomenon, remains to be determined.

In summary, this is the first B-cell epitope mapping of the autoantigen AQP4. A proportion of anti-AQP4 antibodies target certain linear epitopes, located in the intracellular domains of the molecule. Our study does not address the conceivable pathogenic role of these specific antibodies but rather raises important questions about the generation of the immune response. One of the 3 epitopes identified in our study, presents high similarity with the human TAX1BP1 protein, which is involved in the pathogenesis of HAM/TSP, a disease that shares common clinical features with neuromyelitis optica. Future studies in our laboratory aim to determine the pathogenic relevance of these findings.

\section{References}

[1] Wingerchuk DM, Lennon VA, Pittock SJ, Lucchinetti CF, Weinshenker BG. Revised diagnostic criteria for neuromyelitis optica. Neurology 2006;66:1485-9.

[2] Jarius S, Paul F, Franciotta D, Waters P, Zipp F, Hohlfeld R, et al. Mechanisms of disease: aquaporin-4 antibodies in neuromyelitis optica. Nat Clin Pract Neurol 2008;4:202-14

[3] Lennon VA, Wingerchuk DM, Kryzer TJ, Pittock SJ, Lucchinetti CF, Fujihara K, et al. A serum autoantibody marker of neuromyelitis optica: distinction from multiple sclerosis. Lancet 2004;364:2106-12.

[4] Wingerchuk DM, Lennon VA, Lucchinetti CF, Pittock SJ, Weinshenker BG. The spectrum of neuromyelitis optica. Lancet Neurol 2007;6:805-15.

[5] Pittock SJ, Lennon VA, de Seze J, Vermersch P, Homburger HA, Wingerchuk DM, et al. Neuromyelitis optica and non organ-specific autoimmunity. Arch Neurol 2008;65:78-83.

[6] Misu T, Fujihara K, Nakashima I, Miyazawa I, Okita N, Takase S, et al. Pure optic-spinal form of multiple sclerosis in Japan. Brain 2002;125:2460-8.

[7] Borgnia M, Nielsen S, Engel A, Agre P. Cellular and molecular biology of the aquaporin water channels. Annu Rev Biochem 1999;68:425-58.

[8] Hiroaki Y, Tani K, Kamegawa A, Gyobu N, Nishikawa K, Suzuki H, et al Implications of the aquaporin-4 structure on array formation and cell adhesion. J Mol Biol 2006;355:628-39.

[9] Lu M, Lee MD, Smith BL, Jung JS, Agre P, Verdijk MA, et al. The human AQP4 gene: definition of the locus encoding two water channel polypeptides in brain. Proc Natl Acad Sci U S A 1996;93:10908-12.

[10] Hinson SR, Pittock SJ, Lucchinetti CF, Roemer SF, Fryer JP, Kryzer TJ, et al. Pathogenic potential of IgG binding to water channel extracellular domain in neuromyelitis optica. Neurology 2007;69:2221-31.

[11] Vincent T, Saikali P, Cayrol R, Roth AD, Bar-Or A, Prat A, et al. Functional consequences of neuromyelitis optica-IgG astrocyte interactions on blood-brain barrier permeability and granulocyte recruitment. Am J Immunol 2008;181:5730-7.

[12] Bradl M, Misu T, Takahashi T, Watanabe M, Mader S, Reindl M, et al. Neuromyelitis optica: pathogenicity of patient immunoglobulin in vivo. Ann Neurol 2009;66:630-43.

[13] Kinoshita M, Nakatsuji Y, Kimura T, Moriya M, Takata K, Okuno T, et al Neuromyelitis optica: passive transfer to rats by human immunoglobulin. Biochem Biophys Res Commun 2009;386:623-7.

[14] Saadoun S, Waters P, Bell BA, Vincent A, Verkman AS, Papadopoulos MC. Intracerebral injection of neuromyelitis optica immunoglobulin $G$ and human complement produces neuromyelitis optica lesions in mice. Brain 2010;133:349-61.

[15] Nicchia GP, Mastrototaro M, Rossi A, Pisani F, Tortorella C, Ruggieri M, et al Aquaporin-4 orthogonal arrays of particles are the target for neuromyelitis optica autoantibodies. Glia 2009;57:1363-73.

[16] Tani T, Sakimura K, Tsujita M, Nakada T, Tanaka M, Nishizawa M, et al. Identification of binding sites for anti-aquaporin 4 antibodies in patients with neuromyelitis optica. J Neuroimmunol 2009;211:110-3.

[17] Marnetto F, Hellias B, Granieri L, Frau J, Patanella AK, Nytrova P, et al. Western blot analysis for the detection of serum antibodies recognizing linear Aquaporin-4 epitopes in patients with Neuromyelitis Optica. J Neuroimmunol; 2009.

[18] Lennon VA, Kryzer TJ, Pittock SJ, Verkman AS, Hinson SR. IgG marker of opticspinal multiple sclerosis binds to the aquaporin- 4 water channel. J Exp Med 2005;202:473-7. 
[19] Hochberg MC. Updating the American College of Rheumatology revised criteria for the classification of systemic lupus erythematosus. Arthritis Rheum 1997;40:1725.

[20] Vitali C, Bombardieri S, Jonsson R, Moutsopoulos HM, Alexander EL Carsons SE, et al. Classification criteria for Sjogren's syndrome: a revised version of the European criteria proposed by the American-European Consensus Group. Ann Rheum Dis 2002;61:554-8.

[21] Routsias JG, Vlachoyiannopoulos PG, Tzioufas AG. Autoantibodies to intracellular autoantigens and their B-cell epitopes: molecular probes to study the autoimmune response. Crit Rev Clin Lab Sci 2006;43:203-48.

[22] Halimi H, Dumortier H, Briand JP, Muller S. Comparison of two different methods using overlapping synthetic peptides for localizing linear B cel epitopes in the U1 snRNP-C autoantigen. J Immunol Methods 1996;199:77-85.

[23] James JA, Harley JB. Linear epitope mapping of an Sm B/B' polypeptide. J Immunol 1992;148:2074-9.

[24] Routsias JG, Tzioufas AG, Sakarellos-Daitsiotis M, Sakarellos C, Moutsopoulos HM. Epitope mapping of the Ro/SSA60KD autoantigen reveals disease-specific antibody-binding profiles. Eur J Clin Invest 1996;26:514-21.

[25] Tzioufas AG, Yiannaki E, Sakarellos-Daitsiotis M, Routsias JG, Sakarellos C Moutsopoulos HM. Fine specificity of autoantibodies to La/SSB: epitope mapping, and characterization. Clin Exp Immunol 1997;108:191-8.

[26] Lundberg K, Kinloch A, Fisher BA, Wegner N, Wait R, Charles P, et al. Antibodies to citrullinated alpha-enolase peptide 1 are specific for rheumatoid arthritis and cross-react with bacterial enolase. Arthritis Rheum 2008;58:3009-19.

[27] Al-Bukhari TA, Radford PM, Bouras G, Davenport C, Trigwell SM, Bottazzo GF, et al. Distinct antigenic features of linear epitopes at the $\mathrm{N}$-terminus and C-terminus of $65 \mathrm{kDa}$ glutamic acid decarboxylase (GAD65): implications for autoantigen modification during pathogenesis. Clin Exp Immunol 2002;130:131-9.

[28] Matiello M, Schaefer-Klein J, Hebrink D, Kingsbury D, Lennon V, Weinshenker B. Genetic analysis of Neuromyelitis Optica: two different Arg19 mutations in the $\mathrm{N}$-terminus of AQP4 suggest a molecular mechanism for susceptibility to NMO. Mult Scler 2009;15:P262.
[29] Saini H, Fernandez G, Kerr D, Levy M. Differential expression of aquaporin-4 isoforms localizes with neuromyelitis optica disease activity. J Neuroimmunol 2010;221:68-72.

[30] Alarcon-Segovia D, Llorente L, Ruiz-Arguelles A. The penetration of autoantibodies into cells may induce tolerance to self by apoptosis of autoreactive lymphocytes and cause autoimmune disease by dysregulation and/or cell damage. J Autoimmun 1996;9:295-300.

[31] Hormigo A, Lieberman F. Nuclear localization of anti-Hu antibody is not associated with in vitro cytotoxicity. J Neuroimmunol 1994;55:205-12.

[32] Lisi S, Sisto M, Soleti R, Saponaro C, Scagliusi P, D’Amore M, et al. Fcgamma receptors mediate internalization of anti-Ro and anti-La autoantibodies from Sjogren's syndrome and apoptosis in human salivary gland cell line A- 253 . J Oral Pathol Med 2007;36:511-23.

[33] Ruiz-Arguelles A, Rivadeneyra-Espinoza L, Alarcon-Segovia D. Antibody penetration into living cells: pathogenic, preventive and immuno-therapeutic implications. Curr Pharm Des 2003;9:1881-7.

[34] Deng SX, Hanson E, Sanz I. In vivo cell penetration and intracellular transport of anti-Sm and anti-La autoantibodies. Int Immunol 2000;12:415-23.

[35] Ehrenstein MR, Katz DR, Griffiths MH, Papadaki L, Winkler TH, Kalden JR, et al. Human IgG anti-DNA antibodies deposit in kidneys and induce proteinuria in SCID mice. Kidney Int 1995;48:705-11.

[36] Nelson P, Khodadoust M, Spencer C, Varrin-Doyer M, Patarroyo Juan C, Prodhomme T, et al. Immunodominant T cell determinants of the autoantigen, aquaporin-4. J Neuroimmunology; 2010:1-219. 10th Course of the European School of Neuroimmunology.

[37] Gachon F, Peleraux A, Thebault S, Dick J, Lemasson I, Devaux C, et al. CREB-2, a cellular CRE-dependent transcription repressor, functions in association with Tax as an activator of the human T-cell leukemia virus type 1 promoter. J Virol 1998;72:8332-7.

[38] Jin DY, Teramoto H, Giam CZ, Chun RF, Gutkind JS, Jeang KT. A human suppressor of c-Jun $\mathrm{N}$-terminal kinase 1 activation by tumor necrosis factor alpha. J Biol Chem 1997;272:25816-23.

[39] Osame M, Usuku K, Izumo S, Ijichi N, Amitani H, Igata A, et al. HTLV-I associated myelopathy, a new clinical entity. Lancet 1986;1:1031-2. 